\title{
Determinação de metais em peixes e \\ camarão-rosa (Farfantepenaeus paulensis) do estuário da Lagoa dos Patos (Pelotas-RS, Brasil) por MIP OES
}

\section{Determination of metals in fish and shrimp (Farfantepenaeus paulensis) from Patos Lagoon (Pelotas- RS, Brazil) by MIP OES}

Sandy Araújo Silva ${ }^{1}$, Daisa Hakbart Bonemann ${ }^{1}$, Josiane dos Santos da Silva ${ }^{1}$, Janaína Garcia Timm¹, Anderson Schwingel Ribeiro ${ }^{1}$, Mariana Antunes Vieira ${ }^{1 *}$

${ }^{1}$ Universidade Federal de Pelotas (UFPel), Centro de Ciências Químicas, Farmacêuticas e Alimentos, Laboratório de Metrologia Química (LabMeQui), Capão do Leão/RS - Brasil

*Corresponding Author: Mariana Antunes Vieira, Universidade Federal de Pelotas (UFPel), Centro de Ciências Químicas, Farmacêuticas e Alimentos, Laboratório de Metrologia Química (LabMeQui), Campus Capão do Leão, s/n, Caixa Postal: 354, CEP: 96160-000, Capão do Leão/RS - Brasil, e-mail: maryanavieira@hotmail.com

Cite as: Silva, S. A., Bonemann, D. H., Silva, J. S., Timm, J. G., Ribeiro, A. S., \& Vieira, M. A. (2019). Determination of metals in fish and shrimp (Farfantepenaeus paulensis) from Patos Lagoon (Pelotas - RS, Brazil) by MIP OES.

Brazilian Journal of Food Technology, 22, e2018049. https://doi.org/10.1590/1981-6723.04918

\section{Resumo}

No presente trabalho, a técnica de espectrometria de emissão óptica com plasma induzido por micro-ondas foi empregada para determinar as concentrações de $\mathrm{Al}, \mathrm{Cd}, \mathrm{Cu}, \mathrm{Hg}, \mathrm{Mg}$, $\mathrm{Sb}$ e $\mathrm{Zn}$ em amostras de peixes (bagre, tainha, traíra, pintado e corvina) e de $\mathrm{Al}, \mathrm{Ba}, \mathrm{Ca}, \mathrm{Cd}, \mathrm{Cr}, \mathrm{Cu}, \mathrm{Fe}, \mathrm{Mg}, \mathrm{Mn}, \mathrm{Ni}, \mathrm{Pb}, \mathrm{Sb}, \mathrm{Ti}$ e $\mathrm{Zn}$ em amostras de camarão-rosa (Farfantepenaeus paulensis). As amostras foram preparadas usando a decomposição ácida com o sistema de refluxo. Para as análises de peixes, fez-se a comparação de dois sistemas de introdução de amostras no plasma: a nebulização convencional e o sistema multimode. Para se obterem as melhores condições utilizando o sistema multimode, parâmetros, como a concentração de $\mathrm{HCl}$ e do agente redutor $\mathrm{NaBH}_{4}$, foram investigados. A exatidão do método foi avaliada através de análises de materiais de referência certificados e de testes de adição de analito. As recuperações obtidas foram satisfatórias para ambas as amostras. Para os peixes, os melhores resultados foram obtidos usando o sistema multimode e as concentrações encontradas (em $\mathrm{mg} \mathrm{kg}^{-1}$ ) foram 6,43 a 24,7 para Al; 0,41 a 6,6 para Cu; 168,2 a 211,2 para Mg, e 23,1 para Zn. Para as amostras de camarão-rosa, as concentrações máximas (em $\mathrm{mg} \mathrm{kg}^{-1}$ ) encontradas foram: 92,1 para Al; 1.070 para Ca; 4,57 para Cu; 87,9 para Fe; 451 para Mg; 8,9 para Mn, e 29,2 para Zn. Todas as concentrações de $\mathrm{Cd}, \mathrm{Cu}, \mathrm{Pb}$ e $\mathrm{Zn}$ encontradas estão dentro dos limites permitidos pela legislação vigente no Brasil, para pescados. Os resultados obtidos mostraram a adequabilidade do método utilizando a técnica de MIP OES para determinação multielementar em amostras de peixe e camarão-rosa.

Palavras-chave: Peixes; Camarão; Metais; MIP OES; Decomposição ácida. 


\begin{abstract}
In the present work, the microwave induced plasma optical emission spectrometry technique was employed to determine the concentrations of $\mathrm{Al}, \mathrm{Cd}, \mathrm{Cu}, \mathrm{Hg}, \mathrm{Mg}, \mathrm{Sb}$ and $\mathrm{Zn}$ in fish samples (catfish, mullet, wolf fish, spotted sorubim and croaker) and $\mathrm{Al}, \mathrm{Ba}, \mathrm{Ca}, \mathrm{Cd}, \mathrm{Cr}, \mathrm{Cu}, \mathrm{Fe}, \mathrm{Mg}, \mathrm{Mn}, \mathrm{Ni}, \mathrm{Pb}, \mathrm{Sb}, \mathrm{Ti}$ and $\mathrm{Zn}$ in shrimp (Farfantepenaeus paulensis) samples. Samples were prepared by acid decomposition with reflux system. For fish analysis, two systems of sample introduction into plasma were compared: conventional nebulization and multimode system. In order to get the best conditions using the multimode system, parameters such as the concentrations of $\mathrm{HCl}$ and of reducing agent $\mathrm{NaBH}_{4}$ were investigated. The method accuracy was assessed through analysis of certified reference materials and analyte addition tests. The obtained recoveries were satisfactory for both samples. For fish, the best results were obtained using the multimode system and the maximum concentrations (in $\mathrm{mg} \cdot \mathrm{kg}^{-1}$ ) were: 6.43 to 24.7 for $\mathrm{Al} ; 0.41$ to 6.6 for $\mathrm{Cu} ; 168.2$ to 211.2 for $\mathrm{Mg}$ and 23.1 for $\mathrm{Zn}$. For shrimp samples, the maximum concentrations (in $\mathrm{mg}^{\mathrm{kg}}{ }^{-1}$ ) were: 92.1 for Al; 1,070 for $\mathrm{Ca} ; 4.57$ for $\mathrm{Cu} ; 87.9$ for Fe; 451 for $\mathrm{Mg} ; 8.9$ for $\mathrm{Mn}$ and $29.2 \mathrm{for} \mathrm{Zn}$. All concentrations found for $\mathrm{Cd}, \mathrm{Cu}, \mathrm{Pb}$ and $\mathrm{Zn}$ are within the limits permitted by the current legislation in Brazil for fish. The results showed the suitability of the present method using MIP OES technique for multielemental determination in fish and shrimp samples.
\end{abstract}

Keywords: Fish; Shrimp; Metals; MIP OES; Acid decomposition.

\title{
1 Introdução
}

Reconhecido como um alimento fundamental em uma dieta equilibrada, os peixes e os frutos do mar são uma excelente fonte de proteínas, vitaminas, minerais e ácidos graxos essenciais (ômega-3) (Olmedo et al., 2013). Dentre os benefícios associados ao seu consumo, destaca-se a redução do risco de doenças cardiovasculares e o auxílio no desenvolvimento neurológico em crianças (Mozaffarian \& Rimm, 2006; Olmedo et al., 2013).

A produção e o consumo de pescados no Brasil vêm crescendo a cada ano. De acordo com o Ministério da Agricultura, Pecuária e do Abastecimento (MAPA), o consumo de pescados no Brasil é de 14,4 kg por habitante por ano, superando o recomendado pela Organização Mundial da Saúde, que é de $12 \mathrm{~kg}$ por habitante por ano. O Relatório da Organização das Nações Unidas para a Alimentação e a Agricultura (FAO) estima que o País registre um crescimento de $104 \%$ na pesca e aquicultura até 2025 , apontando, desta forma, um incremento neste setor (Brasil, 2017).

Os peixes, por serem consumidores e pertencerem ao nível superior da cadeia alimentar aquática, podem acumular poluentes persistentes (Mansour \& Sidky, 2002; Karadede et al., 2004). Dados científicos indicam que os peixes são, potencialmente, as principais fontes da exposição humana a vários poluentes ambientais, tais como os metais tóxicos. Esses compostos estão naturalmente presentes em ambientes aquáticos e chegam através de vários processos geoquímicos ou fontes antropogênicas, como as águas industriais e agrícolas, e os esgotos urbanos (Chahid et al., 2014).

Uma das características dos metais é a falta da biodegradabilidade. Quando inseridos no ambiente aquático, são redistribuídos em toda coluna de água, sendo depositados ou acumulados em sedimentos e consumidos pela biota (fitoplâncton, zooplâncton, comunidades bentônicas, peixes e demais vertebrados aquáticos). Logo, os peixes são também considerados bons indicadores de poluição aquática (Makedonski et al., 2017; Bosch et al., 2016).

Alguns metais, em quantidades mínimas, desempenham funções importantes no metabolismo dos seres vivos, sendo estes chamados de metais essenciais, como $\mathrm{Ca}, \mathrm{Cu}, \mathrm{Fe}, \mathrm{Mg}, \mathrm{Na}, \mathrm{Zn}$, etc. Outros, diversamente, não apresentam função biológica relevante, podendo causar danos ao metabolismo, sendo estes chamados de metais não essenciais, como Al, Cd, Hg, Pb, etc. (Lima \& Merçon, 2011; Carmo et al., 2011). 
No Brasil, o controle da quantidade de resíduos contaminantes em alimentos de origem vegetal e animal é realizado pelo MAPA. O Plano Nacional de Controle de Resíduos e Contaminantes (PNCRC) é um programa federal de inspeção e fiscalização das cadeias produtivas de alimentos que tem como objetivo realizar o monitoramento da presença de resíduos de substâncias químicas potencialmente nocivas à saúde do consumidor, incluindo contaminantes orgânicos e inorgânicos. Para pescados e crustáceos, a Instrução Normativa n. ${ }^{\circ} 13$ de 15 de julho de 2015 divulgou o subprograma de monitoramento do PNCRC (Brasil, 2015), destacando os limites de referências para os contaminantes inorgânicos $\mathrm{As}, \mathrm{Cd}, \mathrm{Pb}$ e $\mathrm{Hg}$, nestes parâmetros: As ( $\left.1.000 \mu \mathrm{g} \mathrm{kg}^{-1}\right)$; Cd ( $0,05 \mathrm{mg} \mathrm{kg}^{-1}$ em pescados e $500 \mu \mathrm{g} \mathrm{kg}^{-1}$ em crustáceos), Pb (300 $\mu \mathrm{g} \mathrm{kg}{ }^{1}$ em pescados e $500 \mu \mathrm{g} \mathrm{kg}^{-1}$ em crustáceos) e $\mathrm{Hg}\left(0,5 \mathrm{mg} \mathrm{kg}^{-1}\right.$ em pescados e $500 \mu \mathrm{g} \mathrm{kg}^{-1}$ em crustáceos $)$. A Agência Nacional de Vigilância Sanitária (ANVISA) também estabelece os limites máximos para contaminantes inorgânicos em pescados e crustáceos através da Resolução n. ${ }^{\circ} 42$ de 29 de agosto de 2013 (Brasil, 2013). Para pescados, os elementos As, $\mathrm{Cd}, \mathrm{Cr}, \mathrm{Cu}, \mathrm{Pb}, \mathrm{Hg}$ e $\mathrm{Zn}$ apresentam limites (em mg kg-1) de 1,$0 ; 0,05 ; 0,1 ; 30 ; 0,30 ; 0,5$ e 50,0, respectivamente. Para os crustáceos, os limites são os mesmos mencionados na Instrução Normativa n. ${ }^{\circ} 13$ (Brasil, 2015).

Os ambientes estuarinos, como, por exemplo, o da Lagoa dos Patos, possuem características de variação de salinidade e elevada concentração de nutrientes e de sedimentos, proporcionando o desenvolvimento de espécies adaptadas às peculiaridades desse ecossistema. No litoral brasileiro, as áreas estuarinas são amplamente utilizadas por comunidades pesqueiras.

Os principais recursos pesqueiros que ocorrem no estuário Lagoa dos Patos são os peixes bagre (Netuma Barba), espécie considerada em colapso e que se alimenta de fragmentos de peixes e de siri; a corvina (Micropogonias furnieri), que se alimenta de algas, poliquetas, pequenos peixes, crustáceos e moluscos; a tainha (Mugil platanus), que se alimenta de detritos, microrganismos bentônicos, bactérias e vegetais em decomposição, e o camarão-rosa (Farfantepenaeus paulensis), espécie com enorme dependência do estuário e o mais importante crustáceo da Lagoa do ponto de vista econômico. Todas estas espécies são ameaçadas com sobrepesca (Vasconcellos \& Haimovici, 2006). Também são encontrados os peixes pintado (Pimelodus clarias), considerado um peixe omnívoro, alimentando-se principalmente de insetos e restos vegetais, e a traíra (Hoplias malabaricus), que é um peixe carnívoro, alimentando-se de insetos, rãs e pequenos peixes (Fischer et al., 2011).

No entanto, a ocupação urbana desordenada e os fortes impactos ambientais atrelados ao desenvolvimento de atividades humanas e industriais, desenvolvidas às margens dos estuários, constituem uma ameaça para esses ambientes. A grande carga de poluentes lançados nos estuários e seus arredores vem diminuindo o estoque pesqueiro, o que reflete negativamente e de forma acentuada nas condições de vida das populações ribeirinhas (Sanches Filho et al., 2013; Paiva et al., 2009). Por essas razões, torna-se importante o desenvolvimento de métodos analíticos com alta sensibilidade e seletividade para determinar as concentrações de metais em peixes e crustáceos, a fim de avaliar o possível risco do seu consumo para a saúde humana (Karadede et al., 2004).

Diante do exposto, o objetivo deste trabalho foi aplicar a técnica de Espectrometria de Emissão Óptica com Plasma Induzido por Micro-ondas (MIP OES), usando diferentes modos de introdução de amostras no plasma, para determinar as concentrações de $\mathrm{Al}, \mathrm{Cd}, \mathrm{Cu}, \mathrm{Hg}, \mathrm{Mg}$, Sb e Zn, em amostras de peixes, e de Al, $\mathrm{Ba}, \mathrm{Ca}, \mathrm{Cd}, \mathrm{Cr}, \mathrm{Cu}, \mathrm{Fe}, \mathrm{Mg}, \mathrm{Mn}, \mathrm{Ni}, \mathrm{Pb}, \mathrm{Sb}$, Ti e Zn, em amostras de camarão-rosa.

\section{Parte experimental}

\subsection{Instrumentação}

As determinações das concentrações dos metais foram realizadas utilizando um espectrômetro de emissão óptica com plasma induzido por micro-ondas da Agilent Technologies, modelo 4200 (Melbourne, Austrália). 
Para a introdução de amostra no MIP OES, foram utilizados dois sistemas: um deles, composto por câmara de nebulização ciclônica tipo duplo passo (nebulização convencional), e o sistema multimode de introdução de amostras (MSIS), que opera de modo simultâneo à nebulização convencional e à geração química de vapor, conforme é apresentado na Figura 1 . O nebulizador de ambos os sistemas foi do tipo OneNeb inerte. O gás utilizado para a manutenção do plasma foi o nitrogênio, obtido do ar atmosférico, a partir de um gerador de nitrogênio da Agilent Technologies, modelo 4107 (Melbourne, Austrália). A vazão de introdução de amostra foi de $2 \mathrm{~mL} \mathrm{~min}^{-1}$, correção de sinal de fundo automático, frequência do magnetron de $2.450 \mathrm{MHz}$, potência aplicada de $1 \mathrm{~kW}$, tempo de leitura de 5 segundos, três replicatas e estabilização de 15 segundos. As condições operacionais para cada analito, como comprimento de onda, posição de observação no plasma e vazão do nebulizador, respectivamente, foram: Al: $396,152 \mathrm{~nm}$; posição -10 e $0,95 \mathrm{~L}$ min $^{-1}$; Ba: 455,403 nm; posição 10 e 0,65 L min-1; Ca: 393,366 nm; posição 10 e $0,6 \mathrm{~L} \mathrm{~min}^{-1}$; Cd: 228,802 nm; posição 10 e $0,55 \mathrm{~L} \mathrm{~min}^{-1}$; $\mathrm{Cr}$ : 425,433 nm; posição -10 e $0,95 \mathrm{~L} \mathrm{~min}^{-1}$; $\mathrm{Cu}: 324,754 \mathrm{~nm}$; posição zero e 0,70 L min ${ }^{-1}$; Fe: 259,993 nm, posição zero, $0,45 \mathrm{~L} \mathrm{~min}^{-1}$; $\mathrm{Hg}$ : 253,652 nm; posição zero e $0,75 \mathrm{~L} \mathrm{~min}^{-1}$; Mg: 285,213 nm; posição 10 e 0,9 L min-1; Mn: 403,076 nm; posição zero, 0,85 L min'-1; Ni: 352,454 nm; posição zero, $0,7 \mathrm{~L} \mathrm{~min}^{-1}$; $\mathrm{Pb}$ : 405,781 nm; posição -10 e $0,70 \mathrm{~L} \mathrm{~min}^{-1}$; $\mathrm{Sb}: 231,147 \mathrm{~nm}$; posição zero e 0,75 L min ${ }^{-1}$; Ti: $334,941 \mathrm{~nm}$; posição 10 e $0,60 \mathrm{~L} \mathrm{~min}^{-1}$, e Zn: $213,875 \mathrm{~nm}$; posição zero e $0,45 \mathrm{~L} \mathrm{~min}^{-1}$.

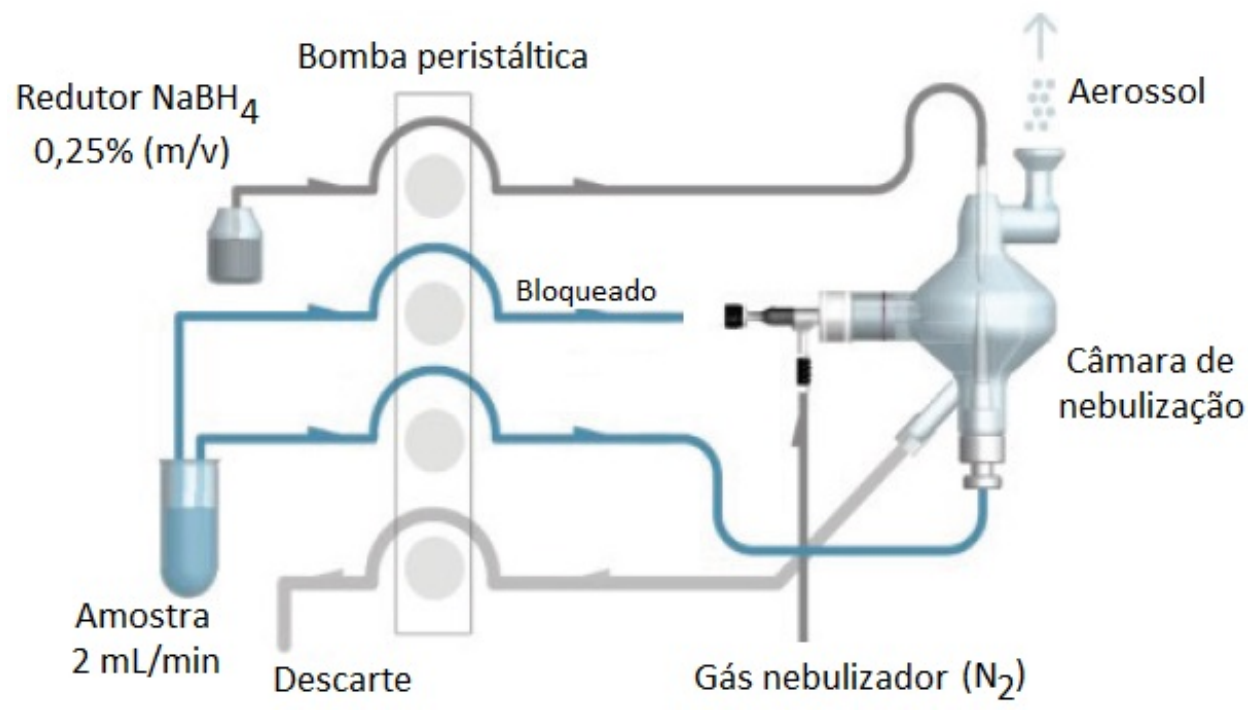

Figura 1. Esquema de funcionamento do sistema multimode em modo duplo. Fonte: Adaptado de Amorin (2016).

Para o preparo das amostras de peixe e camarão, utilizou-se um bloco digestor convencional modelo MA-4025 (Marconi, Brasil). Aos tubos de digestão, foram acoplados os sistemas de refluxo (dedo frio) com um encaixe de Teflon, que apresenta uma ranhura lateral para alívio de pressão. O sistema citado tem circulação interna de água com temperatura controlada de $15^{\circ} \mathrm{C}$ através de um banho termostatizado modelo MA-083 (Marconi, Brasil). Maiores informações sobre o sistema podem ser encontradas conforme descrito por Oreste et al. (2013).

\subsection{Reagentes e amostras}

Os reagentes utilizados foram todos de grau analítico. Para o preparo das soluções, água deionizada obtida a partir de um sistema de destilação de vidro modelo MA078/5 (Marconi, Brasil) e posteriormente eluída em um desionizador modelo CS1800 Evolution (Permution, Brasil) foi utilizada. Os ácidos utilizados foram $\mathrm{HNO}_{3} 65 \%(\mathrm{~m} / \mathrm{m})$ (Vetec, Brasil) e $\mathrm{HCl} 37 \%(\mathrm{~m} / \mathrm{m})$ (Química Moderna, Brasil). Ambos foram bidestilados em um destilador de quartzo MA-075 (Marconi, Brasil). Foi utilizado também $\mathrm{H}_{2} \mathrm{O}_{2} 35 \%(\mathrm{~m} / \mathrm{m})$ (Dinâmica, Brasil). A solução redutora foi preparada a partir do $\mathrm{NaBH}_{4}$ 96,0\% (Fluka, Alemanha) e estabilizada com 
$\mathrm{NaOH} 99,0 \%$ (Vetec, Brasil). As soluções padrão foram preparadas a partir da solução padrão multielementar contendo $100 \mathrm{mg} \mathrm{L}^{-1}$ de cada analito (Sigma-Aldrich, Suíça), com exceção do Hg, em que foi utilizada uma solução estoque monoelementar contendo $1.000 \mathrm{mg} \mathrm{L}^{-1}$ (Fluka, Suíça). A exatidão foi avaliada usando materiais de referência certificados: TORT-2 (hepatopâncreas de lagosta), DORM-3 (músculo de peixe) e DOLT-4 (fígado de peixe) ambos do National Research Council Canada (NRC); SRM 2976 (tecido de mexilhão) e SRM 1546 (homogeneizado de carne), ambos do National Institute of Standards and Technology (NIST), e também com testes de adição e recuperação do analito. Os padrões para as curvas de calibração foram preparados em meio de $\mathrm{HNO}_{3} 2,2 \%(\mathrm{v} / \mathrm{v})$.

As amostras de peixes incluindo diferentes espécies, como bagre (Netuma Barba), tainha (Mugil platanus), pintado (Pimelodus maculatos), traíra (Hoplias malabaricus) e corvina (Micropogonias furnieri), foram adquiridas no Mercado Público de Pelotas (cerca de três a quatro peixes de cada espécie). As amostras de camarão-rosa (da espécie Farfantepenaeus paulensis) foram adquiridas em diferentes locais: na colônia de pescadores Z-3 em Pelotas-RS (Z3/RS), na comunidade de pescadores de São José do Norte-RS (SJN/RS) e na comunidade de pescadores de Palhoça-SC (PL/SC). As amostras de peixes foram adquiridas in natura na forma de filés (limpos). Os camarões foram limpos, descartando a cabeça e a casca. Após a etapa de limpeza, cada amostra foi triturada em um processador doméstico para melhor homogeneização e todas foram congeladas a $-16{ }^{\circ} \mathrm{C}$ até o momento do preparo da amostra para as análises.

\subsection{Métodos de preparo de amostra}

Para o preparo das amostras de peixes, utilizou-se o método que foi desenvolvido por Alves et al. (2018). Foi pesado aproximadamente $0,25 \mathrm{~g}$ de amostras de filé de peixe diretamente nos tubos de digestão e, posteriormente, adicionaram-se 5,0 $\mathrm{mL}$ de $\mathrm{HNO}_{3} 65,0 \%$ (v/v). Em seguida, o sistema de refluxo foi acoplado aos tubos de digestão e, então, os tubos foram levados ao aquecimento no bloco digestor por duas horas a $150^{\circ} \mathrm{C}$. Passados três quartos do tempo de decomposição previsto, adicionaram-se aos tubos $2,0 \mathrm{~mL}$ de $\mathrm{H}_{2} \mathrm{O}_{2}$, e deixou-se por mais 30 minutos no bloco digestor. Após o resfriamento, a solução resultante foi transferida de forma quantitativa para frascos volumétricos de polipropileno e avolumadas a $50,0 \mathrm{~mL}$ com água deionizada.

Para as amostras de camarão, foi pesado $1,5 \mathrm{~g}$ de amostra in natura nos tubos de digestão e adicionados 5,0 $\mathrm{mL}$ de $\mathrm{HNO}_{3} 65,0 \%$ (v/v). Foi utilizado o mesmo sistema mencionado anteriormente e as amostras permaneceram por uma hora e 30 minutos a $120^{\circ} \mathrm{C}$ no bloco digestor. Transcorrido este tempo, adicionou-se $1,40 \mathrm{~mL}$ de $\mathrm{H}_{2} \mathrm{O}_{2}$ e deixou-se em aquecimento por mais 30 minutos. Após o resfriamento, a solução resultante foi transferida para frascos de polipropileno e avolumada a $50,0 \mathrm{~mL}$ com água deionizada.

\section{Resultados e discussão}

\subsection{Determinação de $\mathrm{Al}, \mathrm{Bi}, \mathrm{Cd}, \mathrm{Cu}, \mathrm{Hg}, \mathrm{Mg}$, Sb, Ti e $\mathrm{Zn}$ em peixes por MIP OES}

\subsubsection{Avaliação dos parâmetros operacionais para o sistema multimode para a análise de peixes}

A Espectrometria de Emissão Óptica com Plasma Induzido por Micro-ondas (MIP OES) é uma técnica multielementar que utiliza o plasma como fonte de energia, sustentado pelo nitrogênio retirado do ar atmosférico, o que a torna bastante vantajosa para análises laboratoriais, sem elevado custo. Essa técnica tem sido empregada para análises de amostras ambientais, de alimentos, etc. Um dos fatores que podem afetar a sensibilidade das técnicas de plasma, como a MIP OES, é o transporte da amostra. Normalmente, os sistemas de introdução convencionais fazem uso de nebulizadores pneumáticos. A técnica de MIP OES possui um sistema que utiliza uma câmara ciclônica modificada, denominada de sistema multimode, como mostrado na Figura 1. A utilização deste sistema tem como objetivo melhorar o transporte para alguns analitos, como os 
formadores de hidreto $(\mathrm{As}, \mathrm{Pb}, \mathrm{Sb}$, etc.) ou de vapor frio $(\mathrm{Hg}, \mathrm{Cd})$, devido à possibilidade de promover a geração química de vapor simultaneamente com a introdução da amostra por nebulização convencional (Amorin, 2016). Assim, neste trabalho, foi realizada uma comparação do sistema de introdução de amostra por nebulização convencional e do sistema multimode para as determinações dos analitos nas amostras de peixes. Assim, alguns parâmetros do sistema multimode, como a concentração do agente redutor $\mathrm{NaBH}_{4} \mathrm{e}$ do carreador $\mathrm{HCl}$, foram avaliados.

$\mathrm{O}$ efeito da concentração do agente redutor $\mathrm{NaBH}_{4}$ foi avaliado em função do sinal de intensidade para os analitos $\mathrm{Al}, \mathrm{Cd}, \mathrm{Cu}, \mathrm{Hg}, \mathrm{Mg}, \mathrm{Sb}$ e $\mathrm{Zn}$, em um intervalo de 0,25 a 2,0\% (m/v), e fixando a concentração de $\mathrm{HCl} \mathrm{em} \mathrm{2,0 \%} \mathrm{(v/v).} \mathrm{Para} \mathrm{estes} \mathrm{estudos,} \mathrm{utilizou-se} \mathrm{uma} \mathrm{solução} \mathrm{padrão} \mathrm{contendo} 25 \mu \mathrm{g} \mathrm{L} \mathrm{L}^{-1}$ de cada analito. Os resultados obtidos estão apresentados na Figura 2, na qual é possível observar que o aumento da concentração de $\mathrm{NaBH}_{4}$ não provocou variações significativas nos sinais de intensidades para os analitos. Para $\mathrm{Cd}, \mathrm{Hg}, \mathrm{Sb}$ e $\mathrm{Zn}$, observou-se um pequeno decréscimo do sinal a partir de 1,0\% (m/v). Este fato pode estar relacionado com a diluição do vapor pelo excesso de gás hidrogênio produzido na reação química dentro do sistema multimode (Amorin, 2016). Assim, foi adotada a concentração de $\mathrm{NaBH}_{4}$ 0,5\% (m/v) em meio de $0,5 \%(\mathrm{~m} / \mathrm{v})$ de $\mathrm{NaOH}$ (valor de concentração de $\mathrm{NaOH}$ recomendado pelo fabricante) para os estudos.
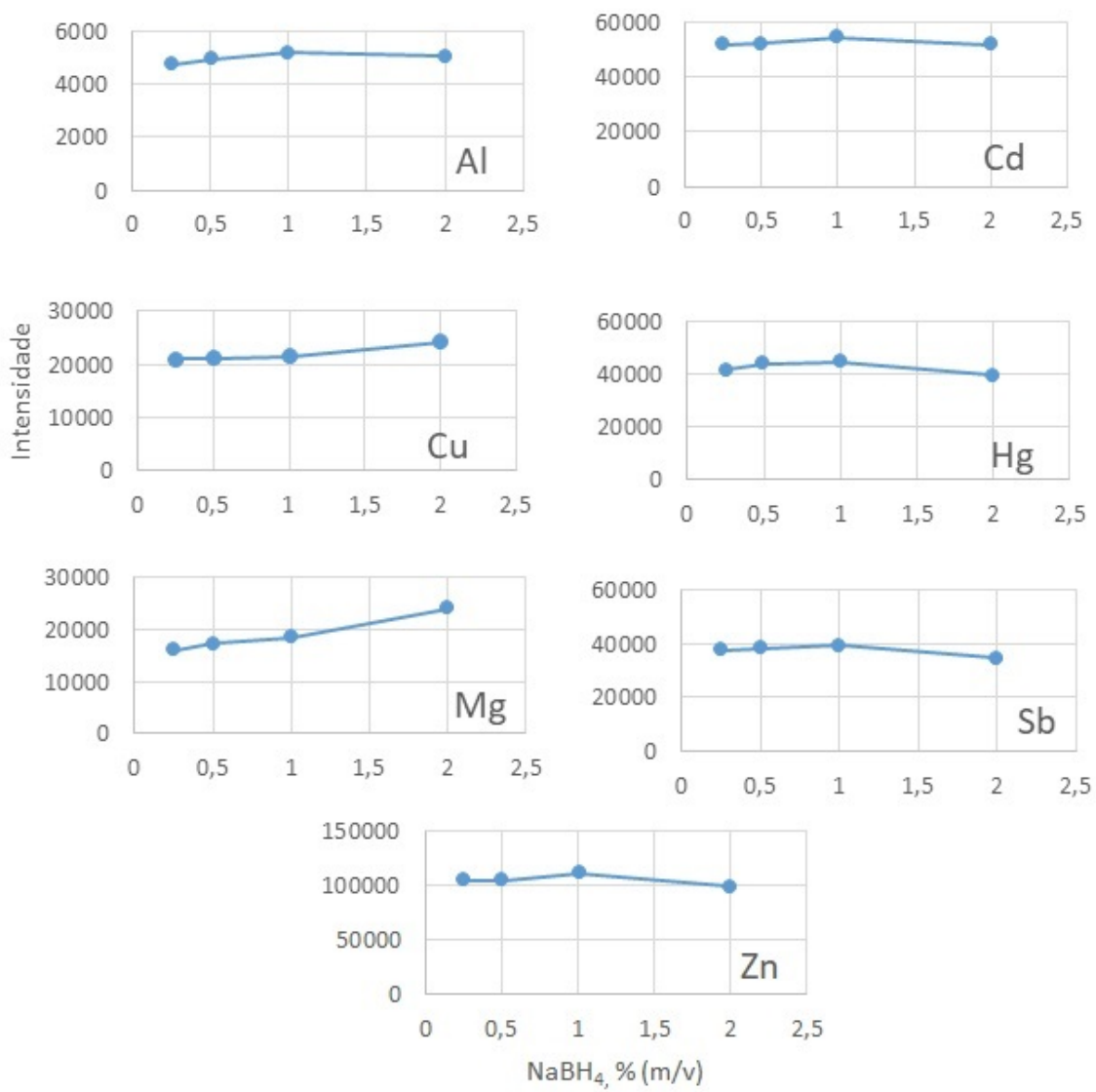

Figura 2. Efeito da concentração de $\mathrm{NaBH}_{4}$ para $\mathrm{Al}, \mathrm{Cd}, \mathrm{Cu}, \mathrm{Hg}, \mathrm{Mg}$, $\mathrm{Sb}$ e $\mathrm{Zn}$ usando o sistema multimode.

Da mesma forma para o $\mathrm{NaBH}_{4}$, o efeito da concentração de $\mathrm{HCl}$ foi avaliado no intervalo de 0,5 a $5,0 \%(\mathrm{v} / \mathrm{v})$ e fixando a concentração de $\mathrm{NaBH}_{4}$ em $0,5 \%(\mathrm{~m} / \mathrm{v})$. De acordo com os resultados apresentados na Figura 3, foi possível observar que também não houve variação significativa nos sinais de intensidades dos analitos em estudo com o aumento da concentração de $\mathrm{HCl}$. Assim, a concentração de $\mathrm{HCl}$ 2,0\% (v/v) foi escolhida, para não haver sobrecarga de ácido na tocha do MIP OES, pois o recomendado pelo fabricante é que se utilizem concentrações de, no máximo, $5 \%$ (v/v). 

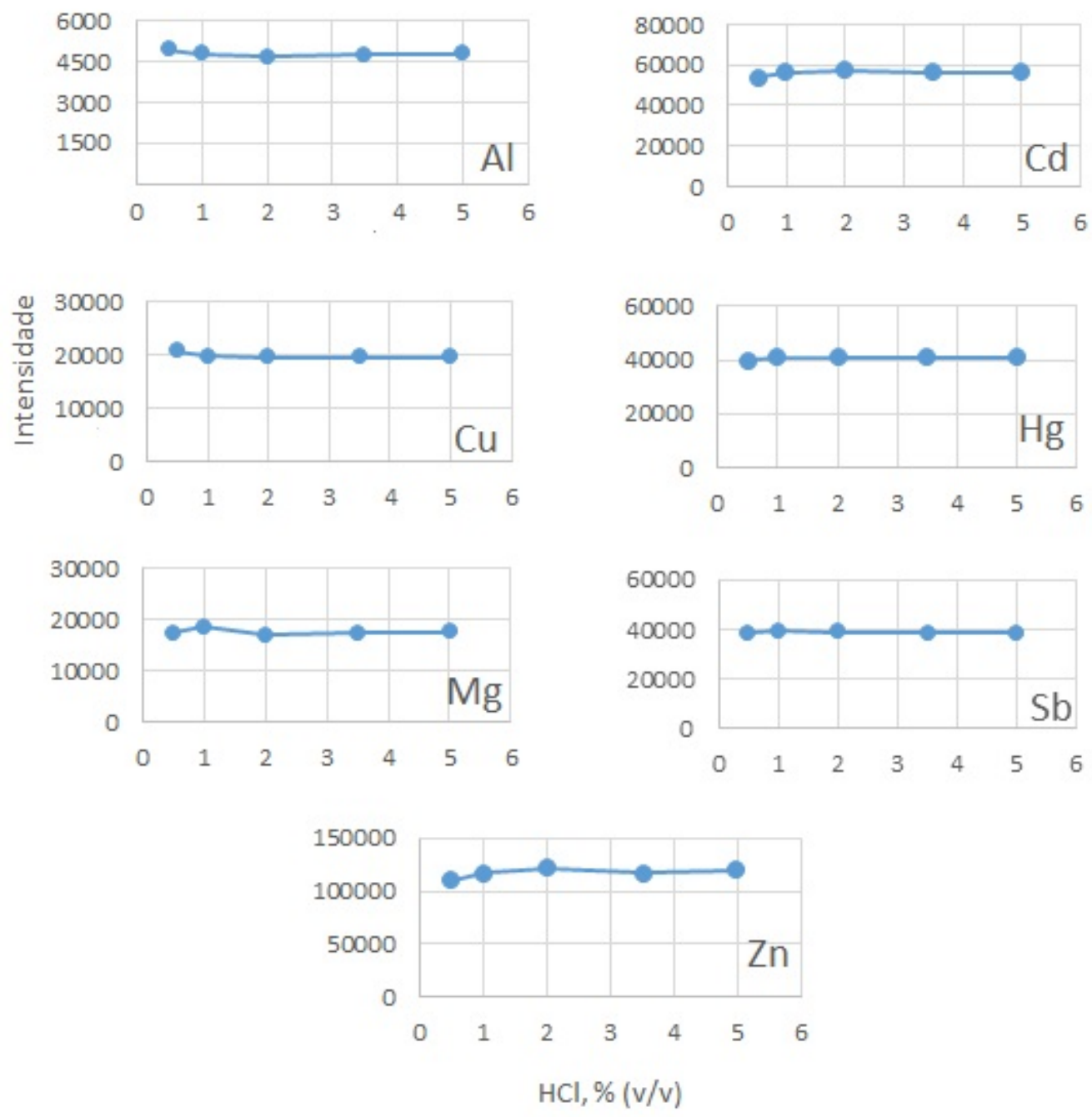

Figura 3. Efeito da concentração de $\mathrm{HCl}$ para $\mathrm{Al}, \mathrm{Cd}, \mathrm{Cu}, \mathrm{Hg}, \mathrm{Mg}$, $\mathrm{Sb}$ e $\mathrm{Zn}$ usando o sistema multimode.

\subsubsection{Parâmetros de mérito e avaliação da exatidão}

Os parâmetros de mérito (Tabela 1), para a determinação das concentrações de $\mathrm{Al}, \mathrm{Cd}, \mathrm{Cu}, \mathrm{Hg}, \mathrm{Mg}$, $\mathrm{Sb}$ e $\mathrm{Zn}$, nas amostras de peixes, foram obtidos a partir dos dados das curvas de calibração e utilizando os dois sistemas de introdução de amostra: a nebulização convencional e o sistema multimode. A faixa linear de calibração empregada para a nebulização convencional foi de 0,1 a $5 \mathrm{mg} \mathrm{L}^{-1} \mathrm{e}$, para o sistema multimode, foi de 10 a $250 \mu \mathrm{g} \mathrm{L}-1$.

Tabela 1. Parâmetros de mérito obtidos pela nebulização convencional e pelo sistema multimode.

\begin{tabular}{ccccc}
\hline \multicolumn{5}{c}{ Nebulização convencional } \\
\hline Analito & $\mathbf{a}\left(\mathbf{L ~ m g}^{-\mathbf{1}}\right)$ & $\mathbf{L D}\left(\mathbf{m g ~ k g}^{-\mathbf{}}\right)$ & $\mathbf{L Q}\left(\mathbf{m g ~ k g}^{-\mathbf{1}}\right)$ & $\mathbf{R}$ \\
\hline $\mathrm{Al}$ & 29100 & 1,2 & 3,9 & 0,999 \\
$\mathrm{Cd}$ & 28960 & 0,2 & 0,8 & 0,999 \\
$\mathrm{Cu}$ & 132806 & 0,2 & 0,6 & 0,999 \\
$\mathrm{Hg}$ & 5045 & 0,6 & 1,9 & 0,971 \\
$\mathrm{Mg}$ & 300263 & 0,05 & 0,2 & 0,999 \\
$\mathrm{Sb}$ & 6447 & 0,5 & 1,7 & 0,999 \\
$\mathrm{Zn}$ & 22178 & 0,1 & 0,3 & 0,999 \\
\hline
\end{tabular}


Tabela 1. Continuação...

\begin{tabular}{|c|c|c|c|c|}
\hline \multicolumn{5}{|c|}{ Sistema multimode } \\
\hline & a $\left(\mathrm{L} \mathrm{mg}^{-1}\right)$ & LD $\left(\mathrm{mg} \mathrm{kg}^{-1}\right)$ & LQ $\left(\mathrm{mg} \mathrm{kg}^{-1}\right)$ & $\mathbf{R}$ \\
\hline $\mathrm{Al}$ & 34577 & 0,04 & 0,13 & 0,999 \\
\hline $\mathrm{Cd}$ & 29464 & 0,19 & 0,64 & 0,999 \\
\hline $\mathrm{Cu}$ & 198600 & 0,01 & 0,05 & 0,999 \\
\hline $\mathrm{Hg}$ & 86502 & 0,01 & 0,05 & 0,999 \\
\hline $\mathrm{Mg}$ & 229700 & 0,01 & 0,01 & 0,999 \\
\hline $\mathrm{Sb}$ & 9700 & 0,6 & 1,9 & 0,996 \\
\hline $\mathrm{Zn}$ & 48400 & 0,01 & 0,03 & 0,999 \\
\hline
\end{tabular}

a: coeficiente angular da curva de calibração; LD: limite de detecção; LQ: limite de quantificação; R: coeficiente de correlação linear.

Através dos resultados dos parâmetros de mérito para ambos os sistemas, é possível observar que as curvas de calibração apresentaram coeficiente de correlação linear maior que 0,99 , indicando a boa linearidade para os dois sistemas de introdução de amostras, com exceção do $\mathrm{Hg}$, que, pelo sistema de nebulização convencional, obteve coeficiente de correlação linear de 0,971. Em relação a outras técnicas multielementares, o MIP OES com sistema multimode permitiu, neste trabalho, alcançar melhores limites de detecção e quantificação. Por exemplo, Sanches Filho et al. (2013), ao avaliarem metais em pescados pela técnica de ICP OES, obtiveram limites de quantificação de 6,92;0,87; 5,30, e 7,40 mg kg${ }^{-1}$ para $\mathrm{Al}, \mathrm{Cd}, \mathrm{Cu}$ e $\mathrm{Zn}$, respectivamente. Também foi observada uma melhora na sensibilidade em relação ao sistema de nebulização convencional, conforme pode ser observado pelos valores dos coeficientes angulares das curvas obtidos, provavelmente em função da geração química de vapor ocorrer simultaneamente, principalmente para os elementos que geram hidretos ( $\mathrm{Sb}$, por exemplo) ou vapor ( $\mathrm{Hg}$ e $\mathrm{Cd}$ ).

Com o intuito de avaliar a exatidão do método para as determinações multielementares em peixes e camarões pela técnica de MIP OES, foram analisados diferentes materiais de referência certificados de origem biológica. Os mesmos foram preparados de acordo com o método usado para as amostras de peixes. Os resultados obtidos estão apresentados na Tabela 2.

Tabela 2. Resultados de concentração obtidos de $\mathrm{Al}, \mathrm{Cd}, \mathrm{Cu}, \mathrm{Fe}, \mathrm{Mg}, \mathrm{Mn}$, Ni e $\mathrm{Zn}$ nos materiais de referência certificados $(\mathrm{n}=3)$.

\begin{tabular}{|c|c|c|c|c|}
\hline & $\begin{array}{c}\text { Valor certificado } \\
\left(\mathrm{mg} \mathrm{kg}^{-1}\right)\end{array}$ & $\begin{array}{c}\text { Valor encontrado } \\
\left(\mathrm{mg} \mathrm{kg}^{-1}\right)\end{array}$ & $\begin{array}{c}\text { DPR } \\
(\%)\end{array}$ & $\begin{array}{c}\text { Recuperação } \\
(\%)\end{array}$ \\
\hline \multicolumn{5}{|c|}{ SRM 2976 (tecido de mexilhão) } \\
\hline $\mathrm{Al}$ & $134 \pm 34$ & $130 \pm 1,3$ & 1,0 & 97,0 \\
\hline \multicolumn{5}{|c|}{ DOLT-4 (fígado de peixe) } \\
\hline $\mathrm{Cd}$ & $24,3 \pm 0,8$ & $21,1 \pm 0,03$ & 0,2 & 87,0 \\
\hline $\mathrm{Cu}$ & $31,2 \pm 1,1$ & $30,7 \pm 0,4$ & 1,3 & 98,4 \\
\hline $\mathrm{Mg}$ & $1500^{*}$ & $1303 \pm 68,2$ & 5,2 & 86,8 \\
\hline \multicolumn{5}{|c|}{ TORT-2 (hepatopâncreas de lagosta) } \\
\hline $\mathrm{Cd}$ & $26,7 \pm 0,6$ & $27,9 \pm 0,2$ & 0,7 & 104,4 \\
\hline $\mathrm{Cu}$ & $106 \pm 10$ & $108,8 \pm 0,2$ & 0,2 & 102,6 \\
\hline $\mathrm{Fe}$ & $105 \pm 13$ & $99,6 \pm 7,9$ & 7,9 & 94,8 \\
\hline $\mathrm{Mn}$ & $13,6 \pm 1,2$ & $13,8 \pm 0,7$ & 5,0 & 101,4 \\
\hline $\mathrm{Ni}$ & $2,5 \pm 0,19$ & $2,3 \pm 0,11$ & 4,8 & 92,0 \\
\hline $\mathrm{Zn}$ & $180 \pm 6,0$ & $181,6 \pm 1,7$ & 0,9 & 100,9 \\
\hline \multicolumn{5}{|c|}{ DORM-3 (músculo de peixe) } \\
\hline $\mathrm{Zn}$ & $51,3 \pm 3,1$ & $42,2 \pm 0,9$ & 2,1 & 82,3 \\
\hline \multicolumn{5}{|c|}{ SRM 1546 (homogeneizado de carne) } \\
\hline $\mathrm{Mg}$ & $163 \pm 11$ & $175,4 \pm 7,6$ & 4,3 & 107,6 \\
\hline
\end{tabular}

*Valor informado; DPR: desvio padrão relativo. 
De acordo com os resultados, observou-se uma boa exatidão do método, pois houve concordância entre os resultados obtidos e os valores certificados ou informados para os analitos, com 95\% de confiança. As recuperações variaram entre $82,3 \%$ e $110,9 \%$, e estão em acordo com os valores de referência aceitáveis pela Association of Official Analytical Chemists (2012), de $80 \%$ a 120\%. Os valores de desvio padrão relativo (DPR) foram menores que 7,9\%, comprovando a boa precisão.

O teste de adição e recuperação do analito também foi aplicado em uma amostra de peixe (corvina) para aqueles elementos que não foram determinados em alguns materiais de referência certificados (por exemplo, devido ao uso de uma massa de amostra elevada). Foram avaliados três níveis de concentrações para ambos os sistemas de introdução de amostras investigados. Para o sistema de nebulização convencional, foram 0,1 ; 0,25 e 0,5 $\mathrm{mg} \mathrm{L}^{-1}$, e para o sistema multimode, 50, 100 e $200 \mu \mathrm{g} \mathrm{L}^{-1}$, considerando a faixa linear empregada para cada analito. Os resultados obtidos estão apresentados nas Tabelas 3 e 4, respectivamente.

Tabela 3. Concentrações obtidas de $\mathrm{Al}, \mathrm{Cd}, \mathrm{Cu}, \mathrm{Mg}$, Sb e Zn por MIP OES utilizando o sistema de nebulização convencional após diferentes adições $(\mathrm{n}=3)$.

\begin{tabular}{|c|c|c|c|c|}
\hline Analito & Sem adição & $0,1 \mathrm{mg} \mathrm{L}^{-1}$ & $0,25 \mathrm{mg} \mathrm{L}^{-1}$ & $0,5 \mathrm{mg} \mathrm{L}^{-1}$ \\
\hline $\mathrm{Al}$ & $<\mathrm{LD}$ & $0,11 \pm 0,01$ & $0,24 \pm 0,01$ & $0,52 \pm 0,01$ \\
\hline $\mathrm{Cd}$ & $<\mathrm{LD}$ & $0,08 \pm 0,01$ & $0,2 \pm 0,01$ & $0,41 \pm 0,01$ \\
\hline $\mathrm{Cu}$ & $<\mathrm{LQ}$ & $0,09 \pm 0,02$ & $0,21 \pm 0,01$ & $0,41 \pm 0,01$ \\
\hline $\mathrm{Mg}$ & $0,33 \pm 0,01$ & $0,42 \pm 0,23$ & $0,55 \pm 0,01$ & $0,76 \pm 0,01$ \\
\hline $\mathrm{Sb}$ & $<\mathrm{LQ}$ & $0,22 \pm 0,01$ & $0,34 \pm 0,01$ & $0,55 \pm 0,01$ \\
\hline $\mathrm{Zn}$ & $<\mathrm{LD}$ & $0,04 \pm 0,01$ & $0,17 \pm 0,01$ & $0,38 \pm 0,01$ \\
\hline
\end{tabular}

LD: limite de detecção; LQ: limite de quantificação.

Com base nos resultados apresentados para o sistema de nebulização convencional (Tabela 3), apenas $\mathrm{Al}, \mathrm{Cd}, \mathrm{Cu}$ e $\mathrm{Mg}$ tiveram boas recuperações, entre 82\% e 110\%. Os analitos $\mathrm{Sb}$ e $\mathrm{Zn}$ tiveram recuperações abaixo dos valores de referência aceitáveis pela AOAC, demonstrando que, para alguns elementos, principalmente os que geram hidretos, o sistema de nebulização convencional não é tão sensível como o sistema multimode. Para $\mathrm{Hg}$, não foi possível recuperar as adições. Já para o sistema multimode (Tabela 4), as recuperações obtidas para os analitos $\mathrm{Hg}$ e Sb ficaram entre 93,1\% e 115,0\%, indicando a boa exatidão do método.

Tabela 4. Concentrações obtidas de Hg e Sb por MIP OES utilizando o sistema multimode após diferentes adições $(\mathrm{n}=3)$.

\begin{tabular}{ccccc}
\hline Analito & Sem adição & $\mathbf{5 0} \boldsymbol{\mu g ~ \mathbf { ~ L } ^ { \mathbf { 1 } }}$ & $\mathbf{1 0 0} \boldsymbol{\mu g ~ \mathbf { ~ L } ^ { - \mathbf { 1 } }}$ & $\mathbf{2 0 0} \boldsymbol{\mu g} \mathbf{~ L}^{\mathbf{1}}$ \\
\hline $\mathrm{Hg}$ & $<\mathrm{LD}$ & $52,7 \pm 0,6$ & $115,7 \pm 0,3$ & $186,2 \pm 0,7$ \\
$\mathrm{Sb}$ & $<\mathrm{LD}$ & $53,4 \pm 0,4$ & $108,7 \pm 1,4$ & $206,6 \pm 10,4$ \\
\hline
\end{tabular}

LD: limite de detecção.

\subsubsection{Resultados de concentração de $\mathrm{Al}, \mathrm{Cd}, \mathrm{Cu}, \mathrm{Hg}, \mathrm{Mg}$, Sb e $\mathrm{Zn}$ em amostras de peixes}

As concentrações de $\mathrm{Al}, \mathrm{Cd}, \mathrm{Cu}, \mathrm{Hg}, \mathrm{Mg}, \mathrm{Sb}$ e $\mathrm{Zn}$ em cinco espécies de peixes típicos do estuário da Lagoa dos Patos foram determinadas utilizando os dois sistemas de introdução de amostras: nebulização convencional e o sistema multimode. Quando se empregou a nebulização convencional, foi possível quantificar apenas o $\mathrm{Mg}$ e as concentrações encontradas (em mg kg-1) foram: 155,5 \pm 8,4 (bagre); 191,5 \pm 8,7 (tainha); 170,7 $\pm 12,6$ (traíra); 167,5 \pm 17 (pintado) e 167,7 $\pm 16,9$ (corvina). Os resultados para os outros elementos ficaram abaixo do limite de detecção do método. Quando o sistema multimode foi usado, foi possível quantificar mais analitos nas amostras e os resultados obtidos estão apresentados na Tabela 5. Os valores de desvio padrão relativo variaram entre $0,1 \%$ e $7,0 \%$, indicando uma boa precisão das medidas. 
Tabela 5. Concentrações de $\mathrm{Al}, \mathrm{Cd}, \mathrm{Cu}, \mathrm{Hg}, \mathrm{Mg}, \mathrm{Sb}, \mathrm{e} \mathrm{Zn}$ em peixes por MIP OES com sistema multimode. Valores em $\mathrm{mg} \mathrm{kg}^{-1} \pm$ desvio padrão $(\mathrm{n}=3)$.

\begin{tabular}{cccccc}
\hline Analito & Bagre & Tainha & Traíra & Pintado & Corvina \\
\hline $\mathrm{Al}$ & $6,43 \pm 0,22$ & $<\mathrm{LQ}$ & $24,7 \pm 0,3$ & $<\mathrm{LQ}$ & $<\mathrm{LD}$ \\
$\mathrm{Cd}$ & $<\mathrm{LQ}$ & $<\mathrm{LD}$ & $<\mathrm{LD}$ & $<\mathrm{LQ}$ & $<\mathrm{LD}$ \\
$\mathrm{Cu}$ & $0,63 \pm 0,07$ & $<\mathrm{LQ}$ & $<\mathrm{LD}$ & $6,6 \pm 0,01$ & $0,41 \pm 0,02$ \\
$\mathrm{Hg}$ & $<\mathrm{LD}$ & $<\mathrm{LD}$ & $<\mathrm{LD}$ & $<\mathrm{LD}$ & $<\mathrm{LD}$ \\
$\mathrm{Mg}$ & $175,9 \pm 5,8$ & $211,2 \pm 14,6$ & $172,6 \pm 1,8$ & $188,6 \pm 0,2$ & $168,2 \pm 1,2$ \\
$\mathrm{Sb}$ & $<\mathrm{LQ}$ & $<\mathrm{LQ}$ & $<\mathrm{LQ}$ & $<\mathrm{LQ}$ & $<\mathrm{LD}$ \\
$\mathrm{Zn}$ & $<\mathrm{LQ}$ & $<\mathrm{LQ}$ & $23,1 \pm 0,1$ & $<\mathrm{LQ}$ & $<\mathrm{LQ}$ \\
\hline
\end{tabular}

LD: limite de detecção; LQ: limite de quantificação.

A aplicação do sistema multimode permitiu quantificar $\mathrm{Al}, \mathrm{Cu}, \mathrm{Mg}$ e $\mathrm{Zn}$ em algumas amostras de peixes. O elemento Al foi detectado nas amostras de bagre e traíra, com concentrações de 6,43 e $24,7 \mathrm{mg} \mathrm{kg}^{-1}$, respectivamente. O valor mínimo encontrado ficou próximo ao relatado por Sanches Filho et al. (2013), em amostra de bagre $\left(6,04 \mathrm{mg} \mathrm{kg}^{-1}\right)$. As concentrações encontradas para $\mathrm{Al}$ neste estudo ficaram abaixo do valor de ingestão média de $30 \mathrm{mg}$ por dia (López et al., 2000; Dantas et al., 2007).

$\mathrm{O}$ elemento $\mathrm{Cu}$ foi detectado nas amostras de bagre, pintado e corvina, com concentrações na faixa de 0,41 a $6,6 \mathrm{mg} \mathrm{kg}^{-1}$. $\mathrm{O}$ valor máximo de $\mathrm{Cu}$ encontrado na amostra de pintado se aproximou ao encontrado por Alves et al. (2018), em peixes da mesma espécie do Canal São Gonçalo, e por Olmedo et al. (2013), em amostras obtidas no mercado público de Granada, Espanha, enquanto que o valor mínimo se aproximou ao encontrado por Grotto et al. (2012). As concentrações de Cu obtidas ficaram todas abaixo do limite máximo permitido para pescados pela ANVISA (Brasil, 2005), de $30 \mathrm{mg} \mathrm{kg}^{-1}$.

Para o Mg, as concentrações variaram de 168,2 a 211,2 $\mathrm{mg} \mathrm{kg}^{-1}$. Apesar de não existir legislação específica para a quantidade de $\mathrm{Mg}$ em alimentos, a ANVISA relata uma ingestão diária recomendada (IDR) de $360 \mathrm{mg}$ por dia para adultos (Brasil, 2005). Grotto et al. (2012) encontraram um valor médio de $265 \mathrm{mg} \mathrm{kg}^{-1} \mathrm{de} \mathrm{Mg}$ em amostras de peixes de rio. Para o $\mathrm{Zn}$, a concentração encontrada foi de $23,1 \mathrm{mg} \mathrm{kg}^{-1}$ apenas no peixe traíra, sendo este valor próximo ao encontrado por Sanches Filho et al. (2013), de $20,97 \mathrm{mg} \mathrm{kg}^{-1}$, e está abaixo do limite máximo permitido, que é de $50 \mathrm{mg} \mathrm{kg}^{-1}$ (Brasil, 2013) em pescados, não indicando alguma contaminação nesta amostra. Para as demais amostras, as concentrações de $\mathrm{Zn}$ ficaram abaixo do LQ.

Como é possível observar, as maiores concentrações de Al e Zn foram encontradas na amostra de traíra. Tais resultados podem ser atribuídos ao fato de que a traíra é um peixe carnívoro, alimenta-se de animais que estão no nível maior na cadeia alimentar aquática, o que indica que as maiores concentrações são resultados do acúmulo de metais em função do fenômeno de biomagnificação (Sanches Filho et al., 2013). Para Cd, Hg e $\mathrm{Sb}$, as concentrações ficaram abaixo do LD ou LQ para todas as amostras investigadas.

\subsection{Determinação multielementar em amostras de camarão-rosa por MIP OES}

\subsubsection{Parâmetros de mérito e avaliação da exatidão}

Os parâmetros de mérito para a determinação de $\mathrm{Al}, \mathrm{Ba}, \mathrm{Ca}, \mathrm{Cd}, \mathrm{Cr}, \mathrm{Cu}, \mathrm{Fe}, \mathrm{Mg}, \mathrm{Mn}, \mathrm{Ni}, \mathrm{Pb}, \mathrm{Sb}, \mathrm{Ti}$ e Zn em amostras de camarão estão apresentados na Tabela 6 . A faixa linear da curva de calibração foi de 0 a $5,0 \mathrm{mg} \mathrm{L}^{-1}$. Os valores dos coeficientes de correlação linear foram maiores que 0,99 , demonstrando a boa linearidade do método.

Tabela 6. Parâmetros de mérito obtidos por MIP OES.

\begin{tabular}{ccccc}
\hline Analito & $\mathbf{a}\left(\mathbf{L}^{-\mathbf{1}} \mathbf{~ m g}\right)$ & $\mathbf{L D}\left(\mathbf{m g ~ k g} \mathbf{~ g}^{-1}\right)$ & $\mathbf{L Q}\left(\mathbf{m g ~ k g}^{-1}\right)$ & $\mathbf{R}$ \\
\hline $\mathrm{Al}$ & 48555 & 0,02 & 0,05 & 0,999 \\
$\mathrm{Ba}$ & 427326 & 0,01 & 0,02 & 0,999 \\
$\mathrm{Ca}$ & 605804 & 0,10 & 0,30 & 0,999 \\
$\mathrm{Cd}$ & 32411 & 0,06 & 0,20 & 0,999
\end{tabular}


Tabela 6. Continuação...

\begin{tabular}{|c|c|c|c|c|}
\hline Analito & a $\left(\mathrm{L}^{-1} \mathrm{mg}\right)$ & LD $\left(m g k^{-1}\right)$ & LQ $\left(\mathrm{mg} \mathrm{kg}^{-1}\right)$ & $\mathbf{R}$ \\
\hline $\mathrm{Cr}$ & 65477 & 0,06 & 0,17 & 0,999 \\
\hline $\mathrm{Cu}$ & 211835 & 0,08 & 0,24 & 0,999 \\
\hline $\mathrm{Fe}$ & 14886 & 0,10 & 0,30 & 0,998 \\
\hline $\mathrm{Mg}$ & 321698 & 0,11 & 0,32 & 0,999 \\
\hline $\mathrm{Mn}$ & 58827 & 0,07 & 0,23 & 0,998 \\
\hline $\mathrm{Ni}$ & 29508,4 & 0,02 & 0,06 & 0,999 \\
\hline $\mathrm{Pb}$ & 16323 & 0,16 & 0,50 & 0,998 \\
\hline $\mathrm{Sb}$ & 8125 & 0,82 & 2,50 & 0,998 \\
\hline $\mathrm{Ti}$ & 47206 & 0,14 & 0,42 & 0,999 \\
\hline $\mathrm{Zn}$ & 20486 & 0,15 & 0,50 & 0,999 \\
\hline
\end{tabular}

a: coeficiente angular da curva de calibração; LD: limite de detecção; LQ: limite de quantificação; R: coeficiente de correlação linear.

De modo a avaliar a exatidão, o teste de adição e recuperação do analito foi aplicado para uma amostra de camarão-rosa, adquirida no mercado público de Pelotas e então limpa. Foi realizada a adição de $1,5 \mathrm{mg} \mathrm{L}^{-1}$ dos analitos investigados e os resultados obtidos, apresentados na Tabela 7, mostraram a boa exatidão do método, com valores de recuperação na faixa de $81 \%$ a $111 \%$.

Tabela 7. Resultados obtidos após adição de $1,5 \mathrm{mg} \mathrm{L}^{-1} \mathrm{em}$ amostra de camarão $(\mathrm{n}=3)$.

\begin{tabular}{cccc}
\hline Analito & Sem adição & Após adição & Recuperação (\%) \\
\hline $\mathrm{Al}$ & $0,06 \pm 0,01$ & $1,61 \pm 0,02$ & 103,0 \\
$\mathrm{Ba}$ & $<\mathrm{LD}$ & $1,59 \pm 0,03$ & 106,0 \\
$\mathrm{Ca}$ & $3,77 \pm 0,27$ & $4,98 \pm 0,59$ & 81,0 \\
$\mathrm{Cd}$ & $<\mathrm{LQ}$ & $1,58 \pm 0,03$ & 105,0 \\
$\mathrm{Cr}$ & $<\mathrm{LD}$ & $1,59 \pm 0,07$ & 106,0 \\
$\mathrm{Cu}$ & $<\mathrm{LD}$ & $1,51 \pm 0,04$ & 101,0 \\
$\mathrm{Fe}$ & $0,14 \pm 0,05$ & $1,53 \pm 0,03$ & 93,0 \\
$\mathrm{Mg}$ & $0,64 \pm 0,01$ & $2,18 \pm 0,06$ & 102,0 \\
$\mathrm{Mn}$ & $<\mathrm{LD}$ & $1,62 \pm 0,06$ & 108,0 \\
$\mathrm{Ni}$ & $<\mathrm{LD}$ & $1,66 \pm 0,04$ & 111,0 \\
$\mathrm{~Pb}$ & $0,16 \pm 0,01$ & $1,53 \pm 0,07$ & 91,0 \\
$\mathrm{Sb}$ & $0,07 \pm 0,02$ & $1,58 \pm 0,02$ & 105,0 \\
$\mathrm{Ti}$ & $<\mathrm{LD}$ & $1,56 \pm 0,02$ & 104,0 \\
$\mathrm{Zn}$ & $<\mathrm{LD}$ & $1,52 \pm 0,04$ & 101,0 \\
\hline
\end{tabular}

LD: limite de detecção; LQ: limite de quantificação.

\subsubsection{Resultados de concentração de $\mathrm{Al}, \mathrm{Ba}, \mathrm{Ca}, \mathrm{Cd}, \mathrm{Cr}, \mathrm{Cu}, \mathrm{Fe}, \mathrm{Mg}, \mathrm{Mn}, \mathrm{Ni}$, Pb, Sb, Ti e Zn em amostras de camarão}

As concentrações obtidas para os analitos investigados nas amostras de camarão-rosa oriundas de três diferentes locais - estuário da Lagoa dos Patos, na qual se situa a colônia Z3, São José do Norte-RS e PalhoçaSC - são apresentadas na Tabela 8. 
Tabela 8. Concentrações de Al, $\mathrm{Ba}, \mathrm{Ca}, \mathrm{Cd}, \mathrm{Cr}, \mathrm{Cu}, \mathrm{Fe}, \mathrm{Mg}, \mathrm{Mn}, \mathrm{Ni}, \mathrm{Pb}, \mathrm{Sb}$, Ti e $\mathrm{Zn}$ em amostras de camarão-rosa por MIP OES $(n=3)$.

\begin{tabular}{|c|c|c|c|}
\hline Analito & Z3/RS (mg kg-1) & SJN/RS (mg kg-1) & PL/SC $\left(\mathrm{mg} \mathrm{kg}^{-1}\right)$ \\
\hline $\mathrm{Al}$ & $<\mathrm{LD}$ & $19,6 \pm 2,1(10,7)$ & $92,1 \pm 7,7(8,4)$ \\
\hline $\mathrm{Ba}$ & $<\mathrm{LD}$ & $<\mathrm{LD}$ & $<\mathrm{LD}$ \\
\hline $\mathrm{Ca}$ & $358 \pm 21,7(6,0)$ & $1056 \pm 25,9(2,4)$ & $1070 \pm 8,8(0,8)$ \\
\hline $\mathrm{Cd}$ & $<\mathrm{LD}$ & $<\mathrm{LD}$ & $<\mathrm{LD}$ \\
\hline $\mathrm{Cr}$ & $<\mathrm{LD}$ & $<\mathrm{LD}$ & $<\mathrm{LD}$ \\
\hline $\mathrm{Cu}$ & $3,02 \pm 0,05(1,6)$ & $4,57 \pm 0,26(5,7)$ & $3,20 \pm 0,11(3,4)$ \\
\hline $\mathrm{Fe}$ & $<\mathrm{LD}$ & $22,4 \pm 1,65(7,4)$ & $87,9 \pm 5,0(5,7)$ \\
\hline $\mathrm{Mg}$ & $371 \pm 8,6(2,3)$ & $389 \pm 21,1(5,4)$ & $451 \pm 14,3(3,2)$ \\
\hline Mn & $0,51 \pm 0,05(9,8)$ & $2,91 \pm 0,27(9,3)$ & $8,9 \pm 0,5(5,6)$ \\
\hline $\mathrm{Ni}$ & $<\mathrm{LD}$ & $<\mathrm{LD}$ & $<\mathrm{LD}$ \\
\hline $\mathrm{Pb}$ & $<\mathrm{LD}$ & $<\mathrm{LD}$ & $<\mathrm{LD}$ \\
\hline $\mathrm{Sb}$ & $<\mathrm{LD}$ & $<\mathrm{LD}$ & $<\mathrm{LD}$ \\
\hline $\mathrm{Ti}$ & $<\mathrm{LD}$ & $<\mathrm{LD}$ & $<\mathrm{LD}$ \\
\hline $\mathrm{Zn}$ & $26,8 \pm 0,1(0,4)$ & $29,2 \pm 0,3(1,0)$ & $26,9 \pm 0,6(2,2)$ \\
\hline
\end{tabular}

LD: limite de detecção.

As amostras de SJN/RS e de PL/SC apresentaram valores de Al e Mn elevados ao compará-los com o índice diário recomendado (IDR), que é de 2,3 mg para o Mn (Brasil, 2005) e de $1 \mathrm{mg} \mathrm{kg}^{-1}$ de peso corpóreo para o Al (World Health Organization, 1996), indicando que estas não estariam aptas para o consumo. Estes elementos provavelmente são oriundos de contaminação pelas práticas agrícolas e pelo descarte inadequado de efluentes doméstico e industrial próximos da região em que são coletadas.

As amostras de PL/SC e SJN/RS apresentaram resultados de concentração para os elementos Ca e Fe maiores que o IDR de 1.000 e $14 \mathrm{mg}$, respectivamente (Brasil, 2005). Para o Cu e Mg, todas as amostras obtiveram IDR acima do valor recomendado ( 0,9 e $260 \mathrm{mg}$, respectivamente) e, para o $\mathrm{Zn}$, os valores obtidos estão abaixo do IDR, que é de $7 \mathrm{mg}$ (Brasil, 2005). As concentrações de $\mathrm{Cu}$ e $\mathrm{Zn}$ em todas as amostras de camarão estão abaixo dos limites permitidos pela legislação (30 mg kg-1 para $\mathrm{Cu}$ e $50 \mathrm{mg} \mathrm{kg}^{-1}$ para $\mathrm{Zn}$ ). Os elementos $\mathrm{Ba}, \mathrm{Cd}, \mathrm{Cr}, \mathrm{Ni}$, $\mathrm{Pb}, \mathrm{Sb}$ e Ti apresentaram concentrações menores que o limite de deteç̧ão do método.

As concentrações de $\mathrm{Mg}$ e $\mathrm{Fe}$ encontradas em nosso trabalho são próximas daquelas reportadas por Grotto et al. (2012), em amostras de crustáceos, e as concentrações de $\mathrm{Cu}$ e $\mathrm{Zn}$ obtidas são próximas àquelas reportadas por Pinto et al. (2013) em amostras de camarão-rosa da cidade de Pelotas.

\section{Conclusão}

A utilização da decomposição ácida com sistema de refluxo como método de preparo das amostras de peixe e de camarão-rosa se mostrou uma boa alternativa para uma análise multielementar e com baixo custo operacional, quando comparado a outras técnicas. O método apresentou boa exatidão e precisão, atestada pela análise de material de referência certificado e testes de adição e recuperação do analito. Os valores de limite de detecção e quantificação obtidos são adequados aos limites máximos permitidos pela legislação brasileira para pescados. Foi possível observar que as concentrações encontradas dos analitos $\mathrm{Cd}, \mathrm{Cu}, \mathrm{Pb}, \mathrm{Hg}$ e Zn nas amostras de peixes bagre, tainha, traíra, pintado e corvina) e de camarão-rosa estão dentro dos limites permitidos pela legislação brasileira para contaminantes, indicando, desta forma, que não há contaminação nestas amostras. Para a maioria dos elementos, com exceção do Al nas amostras de camarão SJN/RS e PL/SC, os valores de concentração encontrados estão em acordo com o valor de ingestão diária recomendada (IDR). Assim, é de suma importância um monitoramento contínuo da concentração de elementos essenciais e não essenciais nos pescados do estuário da Lagoa dos Patos, de modo a assegurar a segurança alimentar, pelo 
fato de que os peixes e camarões deste local servem como fonte de renda e também são consumidos pela população local.

\section{Agradecimentos}

Os autores agradecem ao Conselho Nacional de Desenvolvimento Científico e Tecnológico (CNPq, Processo n. ${ }^{\circ}$ 310917/2013-1) pela bolsa de produtividade de M. A. Vieira. Agradecem também à Fundação de Amparo à Pesquisa do Rio Grande do Sul (FAPERGS) pelo suporte financeiro, através do Projeto aprovado no Edital n. ${ }^{\circ}$ 001/2013 - Programa Pesquisador Gaúcho - PqG (2027-2551/13-3SIAFEM).

\section{Referências}

Alves, M. M., Medina, A. L., Pinto, A. M. T., Antunes, A. C. N., Sanches Filho, P. J., Ribeiro, A. S., \& Vieira, M. A. (2018). Evaluation of the concentration of $\mathrm{Cu}, \mathrm{Zn}, \mathrm{Pb}$ and $\mathrm{Cr}$ in different fish species from the São Gonçalo channel in Pelotas-RS, Brazil. Journal of the Brazilian Chemical Society, 29(2), 285-296. http://dx.doi.org/10.21577/0103-5053.20170139.

Amorin, A. C. (2016). Use of the Agilent Multimode Sample Introduction System (MSIS) for Simultaneous Hydride Determination and Conventional Nebulization Using the PerkinEImer Optima 7/8x00 Series ICP-OES Systems. Application note. Califórnia: Agilent Technologies, Inc. Recuperado em 30 de junho de 2018, de https://www.agilent.com/cs/library/applications/59916509EN.pdf

Association of Official Analytical Chemists - AOAC. (2012). AOAC Official methods of analysis. Appendix F: Guidelines for Standard Method Performance Requirements. Washington: AOAC.

Bosch, A. C., O'Neill, B., Sigge, G. O., Kerwath, S. E., \& Hoffman, L. C. (2016). Heavy metals in marine fish meat and consumer health: a review. Journal of the Science of Food and Agriculture, 96(1), 32-48. PMid:26238481.

http://dx.doi.org/10.1002/jsfa.7360

Brasil. Ministério da Saúde. Agência Nacional de Vigilância Sanitária. (2005, setembro 22). Dispõe sobre o Regulamento técnico sobre a ingestão diária recomendada (IDR) de proteína, vitaminas e minerais (Resolução RDC ${ }^{\circ} 269$, de 22 de setembro de 2005). Diário Oficial [da] República Federativa do Brasil, Brasília. Recuperado em 30 de junho de 2018, de http://bvsms.saude.gov.br/bvs/saudelegis/anvisa/2005/rdc0269_22_09_2005.html

Brasil. Ministério da Saúde. Agência Nacional de Vigilância Sanitária. (2013). Dispõe sobre o Regulamento Técnico MERCOSUL sobre Limites Máximos de Contaminantes Inorgânicos em Alimentos (Resolução RDC n 42, de 29 de agosto de 2013). Diário Oficial [da] República Federativa do Brasil, Brasília. Recuperado em 30 de junho de 2018, de http://bvsms.saude.gov.br/bvs/saudelegis/anvisa/2013/rdc0042_29_08_2013.pdf

Brasil. Ministério da Agricultura, Pecuária e Abastecimento. (2015, Julho 20). Plano Nacional de Controle de Resíduos e Contaminantes - PNCRC de 2015 para as cadeias de carnes bovina, suína, caprina, ovina, equina, coelho, aves, avestruz, de leite, pescado, mel e ovos (Instrução Normativa n 13, de 15 de julho de 2015). Diário Oficial [da] República Federativa do Brasil, Brasília. Recuperado em 30 de junho de 2018, de http://www.agricultura.gov.br/assuntos/inspecao/produtosanimal/plano-de-nacional-de-controle-de-residuos-e-contaminantes/documentos-da-pncrc/pncrc-2015.pdf

Brasil. (2017, janeiro). Produção de peixes no Brasil cresce com apoio de pesquisas da Embrapa. Governo do Brasil. Recuperado em 30 de junho de 2018, de http://www.brasil.gov.br/economia-e-emprego/2017/01/producao-de-peixes-no-brasilcresce-com-apoio-de-pesquisas-da-embrapa

Carmo, C. A., Abessa, D. M. S., \& Machado Neto, J. G. (2011). Metais em águas, sedimentos e peixes coletados no estuário de São Vicente-SP, Brasil. O Mundo da Saúde, 35(1), 64-70. Recuperado em 30 de junho de 2018, de http://bvsms.saude.gov.br/bvs/artigos/metais_aguas_sedimentos_peixes_estuario_sao_vicente\%20.pdf

Chahid, A., Hilali, M., Benlhachimi, A., \& Bouzid, T. (2014). Contents of cadmium, mercury and lead in fish from the Atlantic sea (Morocco) determined by atomic absorption spectrometry. Food Chemistry, 147, 357-360. PMid:24206730. http://dx.doi.org/10.1016/j.foodchem.2013.10.008

Dantas, S. T., Saron, E. S., Dantas, F. B. H., Yamashita, D. M., \& Kiyataka, P. H. M. (2007). Determinação da dissolução de alumínio durante cozimento de alimentos em panelas de alumínio. Food Science and Technology (Campinas), 27(2), $291-297$. http://dx.doi.org/10.1590/S0101-20612007000200014

Fischer, L. G., Pereira, L. E. D., \& Vieira, J. P. (2011). Peixes estuarinos e costeiros (2. ed.). Rio Grande: Luciano Gomes Fischer.

Grotto, D., Batista, B. L., Carneiro, M. F. H., \& Barbosa Junior, F. (2012). Evaluation by ICP-MS of essential, nonessential and toxic elements in Brazilian fish and seafood samples. Food and Nutrition Sciences, 3(9), 1252-1260. http://dx.doi.org/10.4236/fns.2012.39165

Karadede, H., Oymakb, S. A., \& Unlü, E. (2004). Heavy metals in mullet, Liza abu, and catfish, from the Ataturk Dam Lake (Euphrates), Turkey. Environment International, 2(30), 183-188. PMid:14749107. http://dx.doi.org/10.1016/S01604120(03)00169-7

Lima, V. F., \& Merçon, F. (2011). Metais pesados no ensino de química. Química Nova na Escola, 33(4), 199-205. Recuperado em 30 de junho de 2018, de http://www.qnesc.sbq.org.br/online/qnesc33_4/199-CCD-7510.pdf 
López, F. F., Cabrera, C., Lorenzo, M. L., \& López, M. C. (2000). Aluminum content in foods and beverages consumed in the Spanish diet. Journal of Food Science, 65(2), 206-210. http://dx.doi.org/10.1111/j.1365-2621.2000.tb15980.x

Mansour, S. A., \& Sidky, M. M. (2002). Ecotoxicological studies. 3. Heavy metals contaminating water and fish from Fayoum Governorate, Egypt. Food Chemistry, 78(1), 15-22. http://dx.doi.org/10.1016/S0308-8146(01)00197-2

Makedonski, L., Peycheva, K., \& Stancheva, M. (2017). Determination of heavy metals in selected black sea fish species. Food Control, 72, 313-318. http://dx.doi.org/10.1016/j.foodcont.2015.08.024

Mozaffarian, D., \& Rimm, E. B. (2006). Fish intake, contaminants, and human health: evaluating the risks and the benefit. Journal of the American Medical Association, 296(15), 1885-1891. PMid:17047219. http://dx.doi.org/10.1001/jama.296.15.1885

Olmedo, P., Hernández, A. F., Pla, A., Femia, P., Navas-Acien, A., \& Gil, F. (2013). Determination of essential elements (copper, manganese, selenium and zinc) in fish and shellfish samples. Risk and nutritional assessment and mercury-selenium balance. Food and Chemical Toxicology, 62, 299-307. PMid:24007738. http://dx.doi.org/10.1016/j.fct.2013.08.076

Oreste, E. Q., Jesus, A., Oliveira, R. M., Silva, M. M., Vieira, M. A., \& Ribeiro, A. S. (2013). New design of cold finger for sample preparation in open system: determination of $\mathrm{Hg}$ in biological samples by CV-AAS. Microchemical Journal, 109, 5-9. http://dx.doi.org/10.1016/j.microc.2012.05.034

Paiva, M. V. C., Silva, J. B., \& Fernandes, J. G. (2009). Estuário do rio Timbó - PE: territorialidade da pesca e impactos ambientais. Revista de Geografia, 26(2), 185-199.

Pinto, A. M. T. P., Hirdes, I. M., \& Sanches Filho, P. J. (2013). Determinação de metais pesados nos camarões (Farfantepenaeus paulensis) consumidos na cidade de Pelotas-RS. Ecotoxicology and Environmental Contamination, 8(1), 129134. https://doi.org/10.5132/eec.2013.01.019.

Sanches Filho, P. J., Fonseca, V. K., \& Holbig, L. (2013). Avaliação de metais em pescado da região do Pontal da Barra, Laguna dos Patos, Pelotas-RS. Ecotoxicology and Environmental Contamination, 8(1), 105-111. https://doi.org/10.5132/eec.2013.01.015.

Vasconcellos, M., \& Haimovici, M. (2006). Status of white croaker Micropogonias furnieri exploited in southern Brazil according to alternative hypotheses of stock discreetness. Fisheries Research, 80(2-3), 196-202.

http://dx.doi.org/10.1016/j.fishres.2006.04.016

World Health Organization - WHO. (1996). Trace elements in human nutrition and health (343 p.). Geneva: WHO. 